\title{
Thyroid-like low-grade nasopharyngeal papillary adenocarcinoma: A case report
}

\author{
TARO HORINO $^{1}$, OSAMU ICHII ${ }^{2}$, KAZU HAMADA-ODE ${ }^{1}$, TATSUKI MATSUMOTO ${ }^{1}$, \\ YOSHIKO SHIMAMURA $^{1}$, KOSUKE INOUE ${ }^{1}$ and YOSHIO TERADA ${ }^{1}$ \\ ${ }^{1}$ Department of Endocrinology, Metabolism and Nephrology, Kochi Medical School, Nankoku, Kochi 783-8505; \\ ${ }^{2}$ Laboratory of Anatomy, Department of Biomedical Sciences, Graduate School of Veterinary Medicine, \\ Hokkaido University, Sapporo, Hokkaido 060-0818, Japan
}

Received December 2, 2015; Accepted September 14, 2016

DOI: $10.3892 /$ mco.2016.1056

\begin{abstract}
Thyroid-like low-grade nasopharyngeal papillary adenocarcinoma (TL-LGNPPA) is a rare neoplasm characterized by morphological analogy to papillary thyroid carcinoma and abnormal expression of thyroid transcription factor-1 (TTF-1). We herein report a rare case of TL-LGNPPA with a review of its clinical, morphological and immunohistochemical characteristics. The patient was a 25 -year-old Japanese woman complaining of a 2-year history of fever of unknown origin. There were no remarkable physical findings and the laboratory tests, including C-reactive protein levels, were normal. Laryngoscopy, magnetic resonance imaging and fluorodeoxyglucose-positron emission tomography identified a pedunculated mass at the roof of the nasopharynx. Histologically, the tumour exhibited papillary growth of cuboidal or columnar epithelium. Tubular architecture and a spindle cell component were also observed focally. Some tumour cells exhibited intranuclear cytoplasmic inclusions. Immunohistochemically, the neoplastic cells were positive for TTF-1, cytokeratin 7 and vimentin, but were negative for thyroglobulin. The Ki-67 labelling index (MIB-1 index) reached 5\% in the most concentrated spot. The patient had neither local recurrence nor distant metastasis 3 years after removal of the tumour. In conclusion, TL-LGNPPA should be included it in the differential diagnosis of fever of unknown origin.
\end{abstract}

Correspondence to: Dr Taro Horino, Department of Endocrinology, Metabolism and Nephrology, Kochi Medical School, Kohasu, Okoh-cho, Nankoku, Kochi 783-8505, Japan

E-mail: horinott@yahoo.co.jp

Abbreviations: TL-LGNPPA, thyroid-like low-grade nasopharyngeal papillary adenocarcinoma; TTF-1, thyroid transcription factor-1

Key words: adenocarcinoma, nasopharynx, thyroid transcription factor-1, fluorodeoxyglucose-positron emission tomography-computed tomography, magnetic resonance imaging

\section{Introduction}

There are several types of nasopharyngeal malignancies, including carcinomas and hematolymphoid, mesenchymal and neurogenic tumours; carcinomas, including non-keratinizing and keratinizing squamous cell types, are the most common type of nasopharyngeal malignancy. Primary nasopharyngeal adenocarcinoma is particularly rare, comprising $<0.5 \%$ of the cases of malignant nasopharyngeal neoplasms. These tumours are generally heterogeneous, displaying various histological subtypes and diverse biological behaviours, although they may be broadly classified into two subtypes, namely the conventional/surface origin-type and the salivary gland-type (1). The former are usually low-grade malignancies with papillary configurations, i.e., low-grade nasopharyngeal papillary adenocarcinomas (LGNPPAs), which likely originate from the nasopharyngeal surface mucosa, whereas the latter include tumours such as mucoepidermoid adenocarcinomas, adenoid cystic carcinomas and polymorphous low-grade adenocarcinomas $(1,2)$. LGNPPA was first described and characterized by Wenig et al in 1988, and since then the published case reports have been scarce (2).

Thyroid-like LGNPPAs (TL-LGNPPAs) represent a small minority of LGNPPAs and they are characterised by abnormal expression of thyroid transcription factor-1 (TTF-1), mimicking papillary thyroid carcinoma. TL-LGNPPA was first described by Carrizo et al in 2005 (3) and, to the best of our knowledge, only 12 cases have been reported to date (3-12). We herein present a novel case of a TL-LGNPPA in a 25 -year-old woman, followed by a brief discussion on this rare entity.

\section{Case report}

A 25-year-old Japanese woman with a 2-year history of fever of unknown origin was referred to the Department of Endocrinology, Metabolism and Nephrology, Kochi Medical School (Nankoku, Japan). There were no remarkable physical findings and the laboratory tests, including C-reactive protein levels, were normal. Thorough diagnostic imaging (Fig. 1A-D), which included magnetic resonance imaging, revealed a $1.7 \times 1.2-\mathrm{cm}$ tumour in the nasopharynx (Fig. 1A and B). To 

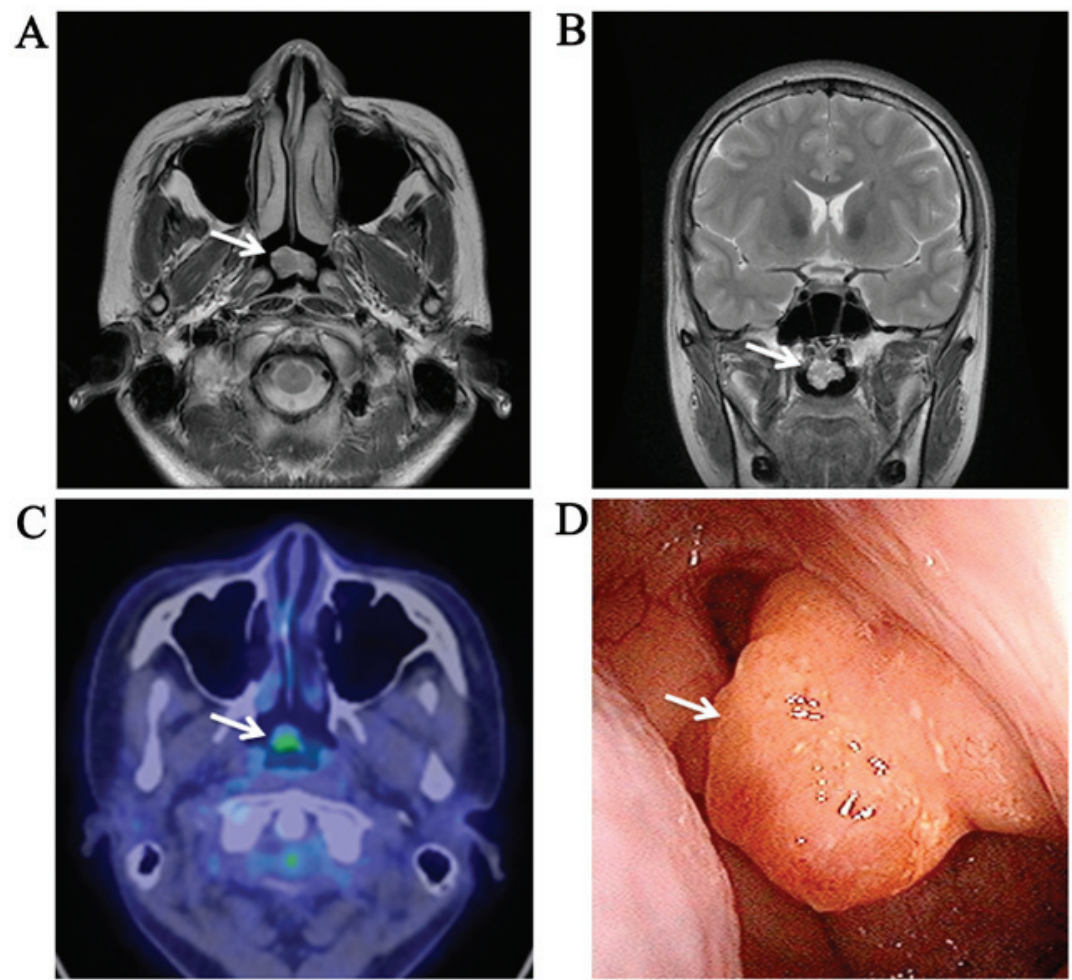

Figure 1. Imaging of the pedunculated mass at the roof of the nasopharynx (arrow). (A) Horizontal and (B) coronal views of the T2-weighted magnetic resonance image. (C) Horizontal view of the ${ }^{18}$ Fludeoxyglucose-positron emission tomography-computed tomography image. (D) Nasopharyngoscopic image of the pedunculated polypoid mass.
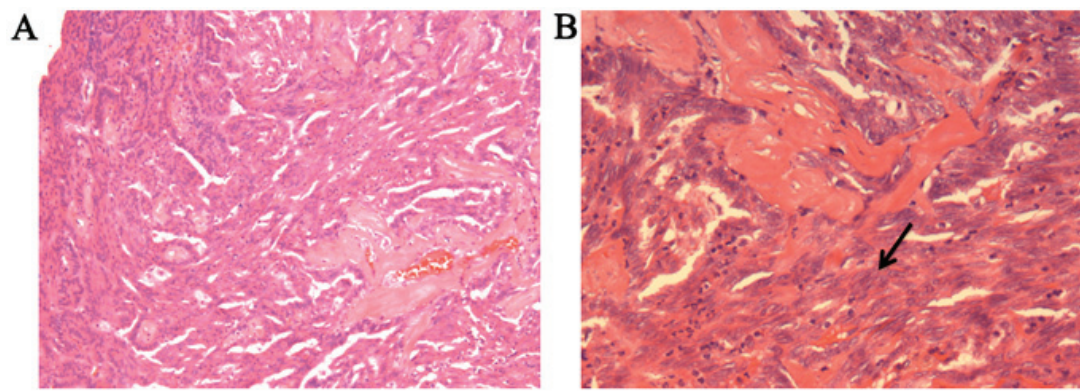

Figure 2. Histological appearance of thyroid-like low-grade nasopharyngeal papillary adenocarcinoma. (A) Tumour cells forming irregular papillary structures with a fibrovascular core. (B) A focal spindle cell component was identified (arrow).

further characterise the tumour, ${ }^{18}$ Fludeoxyglucose-positron emission tomography-computed tomography was performed, and the tumour displayed abnormal uptake and accumulation of the tracer (Fig. 1C). The cervical lymph nodes and thyroid gland were checked by computed tomography and ultrasonography, and no abnormalities were detected. Thyroid function tests confirmed that the patient was euthyroid. Further systemic radiological imaging studies confirmed that there were no metastatic lesions. On laryngoscopy, the tumour was described as a pedunculated mass arising from the roof of the nasopharynx (Fig. 1D). Finally, the patient underwent complete resection of the tumour.

The histopathological examination confirmed negative margins. Microscopically, the tumour exhibited a papillary configuration with fibrovascular cores (Fig. 2A). Each papilla was covered with cuboidal or columnar epithelial cells containing round to ovoid nuclei. There were foci of tubular architecture, and a spindle cell component was observed (Fig. 2B). On immunohistochemical examination, the tumour cells were diffusely positive for TTF-1 (Fig. 3A), whereas they were negative for other thyroid-related proteins, including thyroglobulin (TG) (Fig. 3D). The neoplastic cells were also positive for cytokeratin (CK)7 (Fig. 3B) and vimentin (Fig. 3C), and negative for CK5/6, CK20, smooth muscle actin, p63 and S-100. The Ki-67 labelling index (MIB-1 index) reached 5\% in the area of greatest concentration. Based on the histological and immunohistochemical findings, the diagnosis of TL-LGNPPA was established.

Two days after surgery, the patient's fever disappeared and she exhibited no other symptoms, so she was discharged from the hospital. Adjuvant therapy was not recommended, and the patient has remained afebrile and free of local recurrence and distant metastasis during the 3 years of postoperative follow-up. 

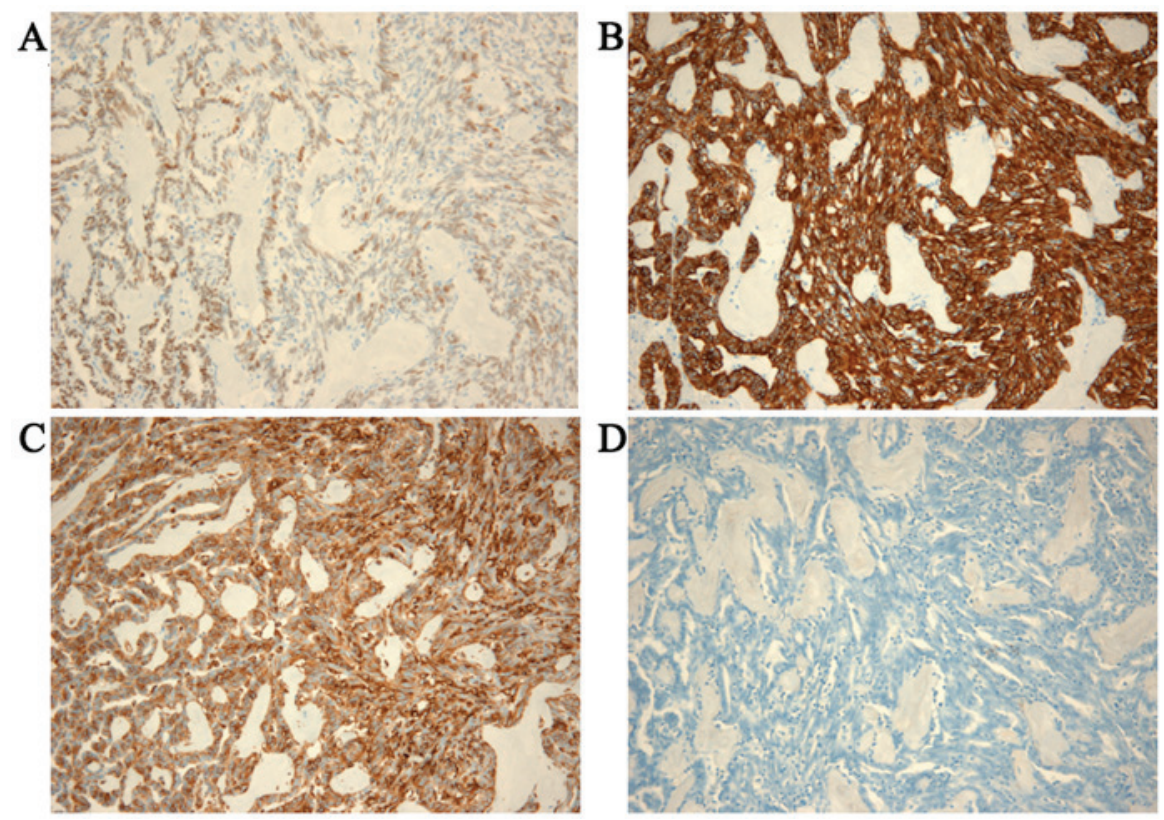

Figure 3. Immunohistochemical staining of the thyroid-like low-grade nasopharyngeal papillary adenocarcinoma. The neoplastic cells were positive for (A) thyroid transcription factor-1, (B) cytokeratin 7 and (C) vimentin, but (D) negative for thyroglobulin.

\section{Discussion}

TL-LGNPPA is an extremely rare neoplasm characterized by morphological analogy to papillary thyroid carcinoma and aberrant TTF-1 expression. Only 12 cases of TL-LGNPPA have been reported to date (3-12). In a review of the clinical characteristics of these tumours described in the previously published cases, Oishi et al (11) reported that the age of the patients ranged from 9 to 68 years (median, 34 years), with a male:female ratio of 1:1. TL-LGNPPA predominantly occurs in the roof of the nasopharynx and at the posterior edge of the nasal septum. The prognosis is excellent, as all cases reported to date have been cured by local excision, whereas local recurrence or metastasis have not been reported in any of the cases. The most common immunohistochemical characteristic of TL-LGNPPA is TTF-1 positivity, which has been noted in every reported case. TL-LGNPPA and papillary thyroid carcinoma share certain immunohistochemical characteristics, such as expression of CK7 and vimentin; however, TL-LGNPPA is usually negative for TG, while papillary thyroid carcinoma is diffusely positive for TG. This finding strongly emphasizes the importance of immunostaining for TG in order to differentiate TL-LGNPPA from papillary thyroid carcinoma. All the findings in our case were compatible with the previously reported clinical and pathological characteristics of TL-LGNPPA.

However, while the majority of the tumours that have been previously reported were incidentally discovered or diagnosed in patients with nasal symptoms including epistaxis and nasal obstruction, our case was diagnosed during a comprehensive evaluation of long-term fever of unknown origin. The central nervous system provides thermoregulatory control of somatic motoneurons, resulting in shivering, and of the sympathetic outflow mediating cutaneous vasoconstriction, which is one of the primary thermoeffector mechanisms conserving heat in the body core and limiting heat loss to the environment; brown adipose tissue, another effector of thermogenesis, acts through parallel, but distinct, effector-specific, integrative/efferent circuits that share common peripheral thermal sensory inputs (13). The hypothalamus is the coordinating or central integration centre for thermoregulation, as it contains the primary integrative and rostral efferent components of these circuits $(13,14)$. The neurons in the hypothalamus, particularly in the preoptic area, are the primary site for the organization and maintenance of febrile response to inflammation and infection, which includes the stimulation of cutaneous vasoconstriction, shivering and brown adipose tissue thermogenesis mediated by prostaglandin E2 on prostaglandin EP3 receptors in the preoptic area (15-18). Pyrogenic cytokines, tumour necrosis factor $\alpha$, interleukin (IL-1 $\beta$ and IL-6 induce cyclooxygenase 2 via the activation pathways of nuclear factor- $\mathrm{\kappa B}$ or signal transducer and activator of transcription 3, leading to prostaglandin E2 synthesis (19). A previous report documented that, in the lung, TTF-1 increased the expression of IL-6, resulting in pulmonary inflammation (20). In our case, although we cannot confirm that the fever was caused by the tumour, we hypothesize that the overexpression of TTF-1 in the tumour may have induced local expression of IL-6, which may have then stimulated the hypothalamus, which was in close proximity to the tumour, thereby leading to fever. As mentioned above, we suspect that TL-LGNPPA in our patient may have been the cause of the fever of unknown origin. However, this theory is poorly evidenced, and it would be interesting to see whether future cases of TL-LGNPPA present in a similar manner.

We herein present a case of TL-LGNPPA in a 25-year-old female patient, exhibiting all the previously reported clinical, morphological and immunohistochemical characteristics of this rare tumour, but presented only as a fever of unknown origin. Our findings and our review of the literature suggest that physicians should be aware of this novel entity and include it in the differential diagnosis of fever of unknown origin. 


\section{References}

1. Barnes L, Eveson JW, Reichart P and Sidransky D: World health organization classification of tumours: Pathology and genetics of head and neck tumors. Lyon: IARC Press, 2005.

2. Wenig BM, Hyams VJ and Heffner DK: Nasopharyngeal papillary adenocarcinoma. A clinicopathologic study of a low-grade carcinoma. Am J Surg Pathol 12: 946-953, 1988.

3. Carrizo F and Luna MA: Thyroid transcription factor-1 expression in thyroid-like nasopharyngeal papillary adenocarcinoma: Report of 2 cases. Ann Diagn Pathol 9: 189-192, 2005.

4. Bansal A, Pradeep KE and Gumparthy KP: An unusual case of low-grade tubulopapillary adenocarcinoma of the sinonasal tract. World J Surg Oncol 6: 54, 2008.

5. Fu CH, Chang KP, Ueng SH, Wu CC and Hao SP: Primary thyroid-like papillary adenocarcinoma of the nasopharynx. Auris Nasus Larynx 35: 579-582, 2008.

6. Ohe C, Sakaida N, Tadokoro C, Fukui H, Asako M, Tomoda K, Tomoda $\mathrm{K}$ and Uemura Y: Thyroid-like low-grade nasopharyngeal papillary adenocarcinoma: Report of two cases. Pathol Int 60: 107-111, 2010.

7. Ozer S, Kayahan B, Cabbarzade C, Bugdayci M, Kosemehmetoglu K and Yucel OT: Thyroid-like papillary adenocarcinoma of the nasopharynx with focal thyroglobulin expression. Pathology 45: 622-624, 2013.

8. Petersson F, Pang B, Loke D, Hao L and Yan B: Biphasic low-grade nasopharyngeal papillary adenocarcinoma with a prominent spindle cell component: Report of a case localized to the posterior nasal septum. Head Neck Pathol 5: 306-313, 2011.

9. Sillings CN, Weathers DR and Delgaudio JM: Thyroid-like papillary adenocarcinoma of the nasopharynx: A case report in a 19-year-old male. Oral Surg Oral Med Oral Pathol Oral Radiol Endod 110: e25-e28, 2010.

10. Wu PY, Huang CC, Chen HK and Chien CY: Adult thyroid-like low-grade nasopharyngeal papillary adenocarcinoma with thyroid transcription factor-1 expression. Otolaryngol Head Neck Surg 137: 837-838, 2007.
11. Oishi N, Kondo T, Nakazawa T, Mochizuki K, Kasai K, Inoue T, Yamamoto T, Watanabe H, Hatsushika K, Masuyama K and Katoh R: Thyroid-like low-grade nasopharyngeal papillary adenocarcinoma: Case report and literature review. Pathol Res Pract 210: 1142-1145, 2014.

12. Huang $\mathrm{CH}$, Chang YL, Wang $\mathrm{CP}$ and Wu HP: Positive immunostaining of thyroid transcription factor-1 in primary nasopharyngeal papillary adenocarcinoma. J Formos Med Assoc 114: 473-474, 2015

13. Morrison SF: Central control of body temperature. F1000Res 5. pii: F1000 Faculty Rev-880, 2016.

14. Tansey EA and Johnson CD: Recent advances in thermoregulation. Adv Physiol Educ 39: 139-148, 2015.

15. Scammell TE, Elmquist JK, Griffin JD and Saper CB Ventromedial preoptic prostaglandin E2 activates fever-producing autonomic pathways. J Neurosci 16: 6246-6254, 1996.

16. Nakamura K, Kaneko T, Yamashita Y, Hasegawa H, Katoh H and Negishi M: Immunohistochemical localization of prostaglandin EP3 receptor in the rat nervous system. J Comp Neurol 42: 543-569, 2000.

17. Nakamura K, Matsumura K, Kaneko T, Kobayashi S, Katoh H and Negishi M: The rostral raphe pallidus nucleus mediates pyrogenic transmission from the preoptic area. J Neurosci 22: 4600-4610, 2002.

18. Lazarus M, Yoshida K, Coppari R, Bass CE, Mochizuki T, Lowell BB and Saper CB: EP3 prostaglandin receptors in the median preoptic nucleus are critical for fever responses. Nat Neurosci 10: 1131-1133, 2007.

19. Inoue W, Somay G, Poole S and Luheshi GN: Immune-to-brain signaling and central prostaglandin E2 synthesis in fasted rats with altered lipopolysaccharide-induced fever. Am J Physiol Regul Integr Comp Physiol 295: R133-R143, 2008.

20. Wert SE, Dey CR, Blair PA, Kimura S and Whitsett JA: Increased expression of thyroid transcription factor-1 (TTF-1) in respiratory epithelial cells inhibits alveolarization and causes pulmonary inflammation. Dev Biol 242: 75-87, 2002. 